\title{
MODELOS FINANCIEROS PARA EL DESARROLLO SOSTENIBLE DE LA ADMINISTRACIÓN PÚBLICA
}

\section{FINANCIAL MODELS \\ FOR THE SUSTAINABLE DEVELOPMENT OF PUBLIC ADMINISTRATION}

\author{
Mario La Torre \\ Universidad de Roma La Sapienza \\ mario.latorre@uniroma1.it \\ Jenny D. Salazar Zapata \\ Universidad de Roma La Sapienza \\ jennydaniela.salazarzapata@uniroma1.it \\ Lorenzo Semplici \\ Ente Nacional Italiano del Microcredito \\ lorenzo.semplici@gmail.com
}




\section{RESUMEN}

Las Administraciones Públicas están cada vez más llamadas a convertirse en protagonistas de la transición hacia el desarrollo sostenible. En esta perspectiva, es necesario pensar en modelos financieros capaces de catalizar recursos públicos y orientarse intencionalmente hacia la implementación de políticas y proyectos funcionales a la transición. Para implementar las políticas públicas de sostenibilidad de manera óptima, no basta la simple referencia a los Objetivos de Desarrollo Sostenible (ODS), que también son una referencia útil y reconocida. Es importante definir estrategias que también definan los métodos de asociación públicoprivada y los de financiamiento de los proyectos de impacto ambiental y social seleccionados. Con esto en mente, el artículo propone un mapeo de las experiencias de finanzas sostenibles en España y en los países de América Latina, clasificándolas según los ODS y las estructuras financieras adoptadas.

Palabras Claves: Objetivos de Desarrollo Sostenible; Finanza Sostenible; Publica Administracion; Proyectos de Impact; Impacto Social; Impacto Ambiental

\section{ABSTRACT}

Public Administrations are increasingly called upon to become protagonists of the transition to sustainable development. In this perspective, it is necessary to think of financial models capable of catalyzing public and private resources, intentionally orienting them towards the implementation of policies and projects functional to the transition. To implement public sustainability policies in an optimal way, the simple reference to the Sustainable Development Goals (SDGs), which are also a useful and recognized reference, is not enough. It is important to define strategies that also define the methods of public-private partnership and those of financing the selected environmental and social impact projects. With this in mind, the article proposes a mapping of sustainable finance experiences in Spain and Latin American countries, classifying them according to the SDGs and financial structures adopted.

Keywords: Sustainable Development Goals; Sustainable Finance; Public Administration; Impact Projects; Social Impact; Environmental Impact

Mario La Torre es profesor titular de "Banca y Finanzas" y "Finanzas Sostenibles y Banca de Impacto" en la Universidad de Roma La Sapienza. Sus principales áreas de investigación son la banca, las finanzas sostenibles y la innovación financiera. También es experto en finanzas audiovisuales y artísticas. Es editor de la serie "Palgrave Studies in Impact Finance". Es responsable del Center for Positive Finance, promotor del University Alliance for Positive Finance, autor del blog Good in Finance.

Jenny Daniela Salazar Zapata es doctoranda en "Banca y Finanzas" en el Departamento de Management de la Universidad de Roma La Sapienza. Sus principales áreas de investigación son la medición del impacto social y medioambiental, las finanzas sostenibles y la integración de la estrategia ESG en modelos de business. Forma parte de los grupos de trabajo nacionales que representan a los jóvenes en la revisión de la Estrategia Nacional de Desarrollo Sostenible en Italia coordinada y promovida por el Ministerio Italiano para la Transición Ecológica.

Lorenzo Semplici, Doctorado en Ciencias de la Economía Civil en LUMSA. Actualmente es responsable del CeSVa (Centro de Estudios y Evaluaciones) de NeXt-Nueva Economía para Todos, colaborador de la Agencia Nacional de Microcréditos e investigador y profesor de la SEC. También colabora en la redacción del Informe sobre los ODS del Foro Nacional del Tercer Sector. Es autor y coautor de varios libros, artículos y documentos de trabajo sobre el bienestar multidimensional, el desarrollo sostenible y la responsabilidad social de las empresas. 


\section{INTRODUCCIÓN}

En la década que comienza, la economía debe situar en el centro de su agenda la transición de actividades, negocios e inversiones que contribuyan a alcanzar los Objetivos del Desarrollo Sostenible (ODS). En cuanto al conjunto de actores sociales y económicos que intervienen en este proceso, el siguiente artículo se centra en la Administración Pública (AP)ำ.

La Comisión Europea, a partir de 2018 con la publicación de un Plan de Acción específico sobre el crecimiento sostenible (European Commission, 2018) hasta el más reciente Recovery Plan (European Commission, 2020), identifica las finanzas sostenibles como una herramienta indispensable para superar la actual crisis sanitaria y, más en general, para alcanzar los Objetivos del Desarrollo Sostenible en el frente social y medioambiental. Además, las instituciones supranacionales (ONU, OCDE) están promoviendo modelos de asociación público-privada para cambiar el enfoque actual de la inversión, asegurándose de que los intereses de los inversores estén alineados con la consecución de los Objetivos de Desarrollo Sostenible de la ONU (OCDE, 2019).

El objetivo de este artículo es ofrecer a los actores del sector público una cartografía de los proyectos de financiación sostenible promovidos por la administración pública en España y en los países de América Latina, útil para inspirar futuras políticas de crecimiento a nivel territorial.

Para aplicar de forma óptima las políticas públicas de sostenibilidad, no basta con remitirse a los Objetivos de Desarrollo Sostenible, que también son una referencia útil y reconocida. Es importante definir estrategias que también definan las modalidades de asociación público-privada y las de financiación de los proyectos seleccionados con impacto ambiental y social.

La peculiaridad de la cartografía propuesta es, por tanto, la de estar construida en base a una doble perspectiva de análisis: los mejores casos se seleccionan, tanto en base a su adhesión a los ODS, como a las arquitecturas financieras utilizadas, inspiradas principalmente en asociaciones público-privadas del tipo pay by result.

Por lo tanto, el artículo contextualiza en primer lugar las dos variables principales sobre las que se desarrolla el mapeo: los Objetivos del Desarrollo Sostenible y las arquitecturas financieras de la financiación sostenible. El apartado dos aclara el vínculo entre los indicadores de desarrollo sostenible y las políticas públicas, y describe el modelo de pago por resultados (pay by results) utilizado por las experiencias más avanzadas de financiación de impacto. La tercera sección propone un mapeo de los proyectos de finanzas sostenibles implementados en la administración pública española y en los países latinoamericanos. Por último, se destacan los rasgos característicos de las experiencias de finanzas sostenibles mapeadas, extrayendo algunas reflexiones para optimizar la integración de las estrategias de sostenibilidad en la AP.

\footnotetext{
${ }^{1}$ El artículo se realiza en el marco del proyecto de investigación "Finanzas sostenibles y administraciones locales: de la teoría a la acción", cofinanciado por la SNA - Scuola Nazionale dell'Amministrazione, en el marco del programa de investigación "Proyectos para una nueva administración pública - Colección de ideas para orientar el cambio en las administraciones públicas" (2020-2021). www.sna.gov.it
} 


\section{SOSTENIBILIDAD EN LA ADMINISTRACIÓN PÚBLICA}

\subsection{LOS OBJETIVOS DE DESARROLLO SOSTENIBLE}

El 25 de septiembre de 2015, la Asamblea General de las Naciones Unidas adoptó la Agenda 2030 en la que se identifican los Objetivos globales cuya consecución puede garantizar un desarrollo económico y social sostenible a largo plazo. La Agenda 2030 consta de 17 objetivos (ODS) que insisten en diferentes ámbitos del desarrollo social y económico. Los objetivos fijados abarcan la pobreza, la desigualdad, el clima, la degradación del medio ambiente, la prosperidad, la paz y la justicia. Pueden agruparse en tres pilares: medioambiental, social y económico (Figura 1).

Figura 1: Objetivos de Desarrollo Sostenible de la ONU en los tres pilares (Stockholm Resilience Centre, 2016)

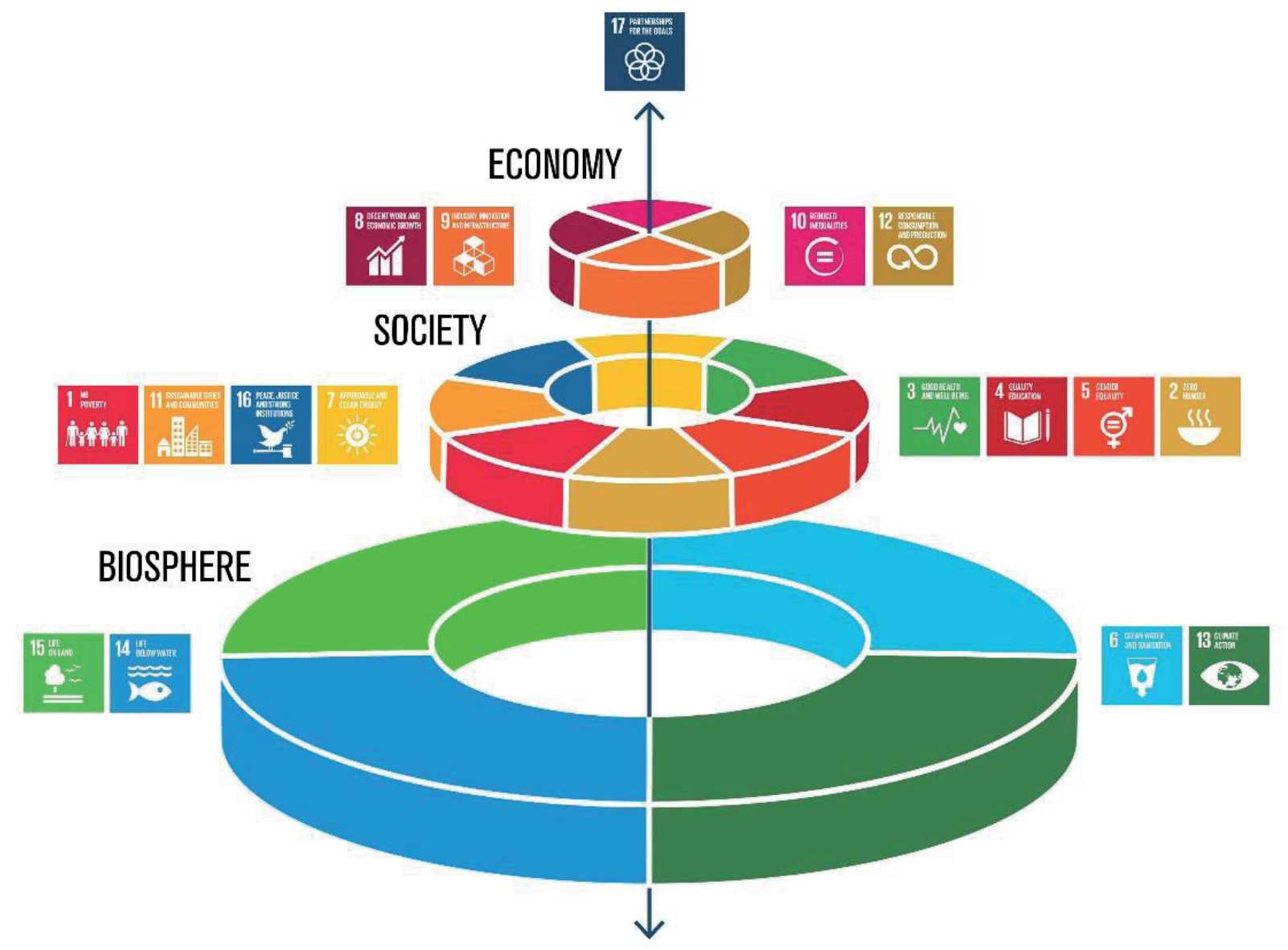

Los 17 objetivos se dividen en 169 sub objetivos (o metas) y el United Inter Agency Expert group on SDGs ha propuesto una lista de más de 230 indicadores necesarios para su seguimiento, que constituyen el marco de referencia a nivel mundial (Department of Economic and Social Affairs - United Nations, 2020). 
La administración pública debe ir necesariamente más allá de la lista desestructurada de proyectos a realizar y financiar, y Europa está impulsando una planificación cuidadosa y coordinada de las políticas y estrategias de sostenibilidad². Por último, los mismos métodos de gestión y distribución de los recursos europeos atribuibles al conocido Recovery Fund, inducen a los Estados miembros a adoptar políticas económicas orientadas a la transición medioambiental, la innovación y la sostenibilidad: cada Estado miembro tendrá que preparar un Plan Nacional de Recuperación y Resiliencia (PNRR), definiendo un programa coherente de reformas e inversiones públicas que se aplicará entre 2021 y 2026.

Para que las administraciones públicas estructuren un plan estratégico de desarrollo sostenible, deben implementar modelos útiles para seleccionar proyectos, iniciativas e inversiones dentro de un marco programático que contemple, por un lado, las prioridades con respecto a los ODS, y por otro, los métodos de financiación de las iniciativas seleccionadas.

Los proyectos públicos inspirados en los ODS, y sus respectivos indicadores de impacto, permiten a las realidades territoriales fijar su propia oferta de bienes, servicios y proyectos en función de las necesidades prioritarias del crecimiento sostenible. Las arquitecturas financieras y las asociaciones permiten enmarcar las inversiones en función de las necesidades de los presupuestos públicos.

En cuanto a la coherencia de las inversiones públicas con los ODS, varias administraciones ya han tomado medidas. Entre ellas se incluyen las Revisiones Locales Voluntarias (o Voluntary Local Reviews $V L R$ ), es decir una herramienta que permite a los gobiernos locales y subnacionales evaluar su logro de los ODS y su contribución a la Agenda 2030. El estudio realizado por el Joint Research Center de la Comisión Europea que monitorea las VLR a nivel europeo evidencia principalmente que: i) hay una importante centralización de la recolección y gestión de datos a nivel nacional; ii) muchas ciudades han podido identificar valiosas fuentes de datos "puramente" locales; iii) después del ODS 11, los objetivos más medidos son $8,4,3$ y 16 .

A modo de ejemplo, en el Ayuntamiento de Madrid el proceso para llegar a la VLR ayudó a la administración local a reforzar la cooperación entre los distintos departamentos del municipio. La Tabla 1 sintetiza el caso de la ciudad de Madrid según los siguientes pasos:

1. clasificación de los servicios en los tres pilares;

2. asociación con los pilares de los ODS;

3. asociación del proyecto específico al objetivo global;

4. identificación de los indicadores de impacto específicos del proyecto.

\footnotetext{
2 "The transition towards climate neutrality can only succeed if we work together, steering our policies in a single direction. Guided by the UN Sustainable Development Goals and the European Green Deal, we can deliver an economy that works for people and the planet" - 26/02/2020 Ursula Von der Leyen Twitter
} 
Tabla 1: Relación entre los proyectos y los Objetivos de Desarrollo Sostenible en la ciudad de Madrid ${ }^{3}$

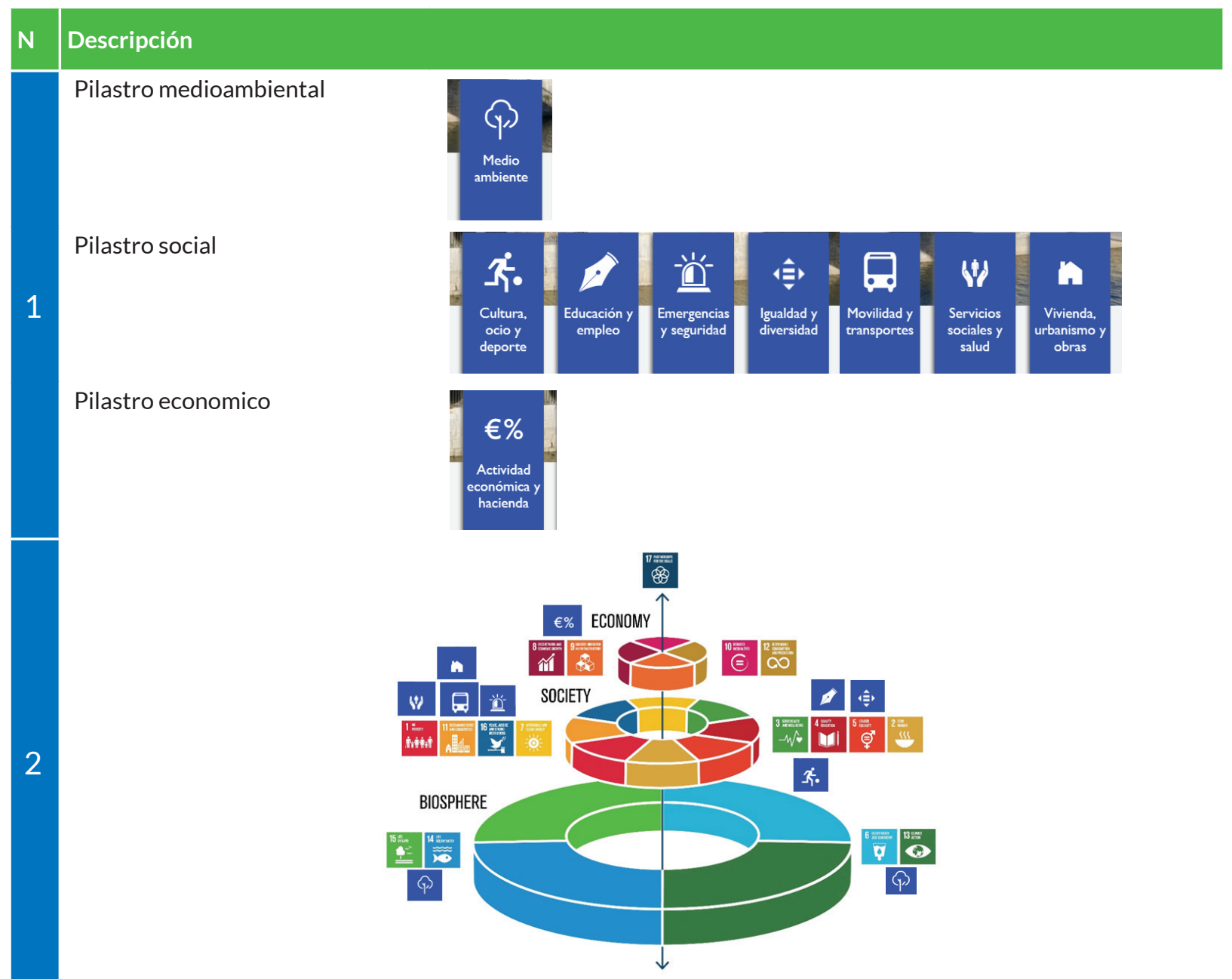

Proyecto de regeneración de agua residual de la ciudad de Madrid a través de 4 plantas Viveros de la Villa, La China, La Gavia y Rejas.

Se posiciona en el servicio de Agua en la sección de servicios sobre el medio ambiente y responde a la Meta 6.4 (Aumentar la eficiencia en el uso del agua) de los ODS.

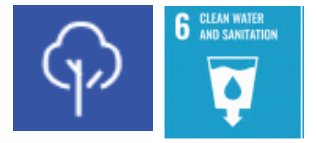

Volumen de agua regenerada en las plantas regeneradoras de agua residual (m3)

Volumen de agua reciclada utilizada para el riego de áreas verdes, zonas deportivas y para la limpieza de otros espacios abiertos como las carreteras.

>> La producción de agua regenerada en las plantas de tratamiento de Madrid corresponde a 6,35 millones de $\mathrm{m}^{3}$ al año. De ellos, el 70\% se utiliza para el riego de zonas verdes públicas, para la limpieza de las calles de la ciudad. El $30 \%$ restante es utilizado por usuarios privados.

Para evaluar el progreso en la eficiencia del uso del agua a lo largo del tiempo, la cantidad total de agua reutilizada debe expresarse como un porcentaje de la cantidad total de agua reciclada.

${ }^{3}$ Servicios a los ciudadanos en la Municipalidad de Madrid. Extraido de: https://www.madrid.es/portal/site/munimadrid 


\subsection{PARTNERSHIP PÚBLICO-PRIVADA (PPP)}

En cuanto a la variable estrictamente financiera - es decir, los modelos de asociación y las arquitecturas financieras dedicadas a los proyectos de sostenibilidad - la AP parece, en cambio, quedarse atrás. La definición que Europa ofrece de las finanzas sostenibles no ayuda al desarrollo de esta perspectiva específica.

Según las indicaciones del Plan de Acción de la Comisión Europea, las finanzas sostenibles se identifican en el proceso de selección y adopción de decisiones de inversión que tienen en cuenta los factores ambientales, sociales y de governance (ESG), con el fin de dirigir los recursos hacia actividades sostenibles con una perspectiva a más largo plazo. La atención se centra, por tanto, en la correlación entre las fuentes de financiación y el tipo de impacto de las inversiones financiadas.

Las finanzas de impacto - la nueva era de las finanzas sostenibles - ofrecen, por el contrario, un interesante campo de pruebas para los modelos financieros funcionales a las políticas de sostenibilidad. Las finanzas de impacto, de hecho, recurren a modelos de asociación público-privada capaces de compensar la falta de recursos públicos, involucrando a inversores privados con diferentes orientaciones en términos de rendimiento, riesgo e impacto. En el contexto del impact investing, las inversiones se inspiran, por definición, en una actitud proactiva hacia el impacto social y medioambiental; el valor social o medioambiental es generado intencionadamente por la inversión y debe, por tanto, ser medible y convertirse en métrica financiera, convirtiéndose en un outcome prioritario. Los inversores se definen como "pacientes" ya que están dispuestos a aceptar rendimientos relacionados con el impacto generado a largo plazo: este es el esquema de remuneración utilizado en los bonos de impacto social (BIS) del tipo pay by results (La Torre, 2017) que difieren de otras formas de bonos sociales (Cuadro 1).

\section{CUADRO 1 - BONO SOCIAL Y BONO VERDE VS BONO DE IMPACTO (SOCIAL/VERDE)}

El Bono Social y el Bono Verde son cualquier tipo de instrumento de bonos - que pueden también ser intercambiados en la bolsa de valores - en donde los ingresos sirven para financiar o refinanciar en parte, o en su totalidad, proyectos sociales nuevos o existentes (por ejemplo un proyecto de social housing). Tipologías de bono social y verde son los "Revenue Bonds", "Project Bonds", y "Social Securitised and covered Bonds" (para más información acerca de las diferencias entre los tres se podría leer el "Social Bond Principles" de ICMA).

Los Bonos de Impacto (Social y Verde) son una arquitectura financiera que se basa sobre un modelo de acuerdo público-privado (public private partnership), que no requiere una emisión en el mercado, en donde los ingresos sirven para financiar proyectos sociales y verdes, y que utiliza un esquema de pay by result.

El impacto generado produce un ahorro en el gasto público que se utiliza para amortizar el capital privado y ofrecer un retorno al inversor que ha adelantado los recursos financieros necesarios para ejecutar la inversión. La asociación público-privada del tipo "pay by result" (PbR) permite promover inversiones sin repercutir en el gasto público: el promotor público encuentra en el inversor privado un financiador que sólo es remunerado cuando se consigue el impacto ambiental y social, y en base al ahorro de gasto público conseguido precisamente por el propio impacto. Esta es, por ejemplo, la estructura utilizada para el bono de impacto social promovido por la ciudad de Buenos Aires (Cuadro 2). 


\section{CUADRO 2- LA PARTNERSHIP PÚBLICO-PRIVADA EN EL BIS: EL CASO DE LA CIUDAD DE BUENOS AIRES}

Proyecta tu Futuro es una iniciativa impulsada y diseñada en 2017 por la Ciudad Autónoma de Buenos Aires (CABA) con el apoyo de dos socios consultores especializados, un equipo de abogados y la asistencia del Fondo Multilateral de Inversiones (FOMIN) y el Banco Interamericano de Desarrollo (BID).

El objetivo principal de Proyectá Tu Futuro es mejorar la tasa de empleo de los jóvenes moderada o extremadamente vulnerables, de entre 17 y 24 años, pertenecientes a la zona sur de la ciudad de Buenos Aires, donde la tasa de desempleo entre los jóvenes es del $24,9 \%$.

Uno de los objetivos que se evalúan para remunerar el bono impacto social es precisamente la finalización del ciclo de estudios por parte de los 728 estudiantes a los que va dirigido.

El siguiente gráfico es representativo de los flujos de capital, los actores implicados y los servicios prestados (Oxford University, 2018).

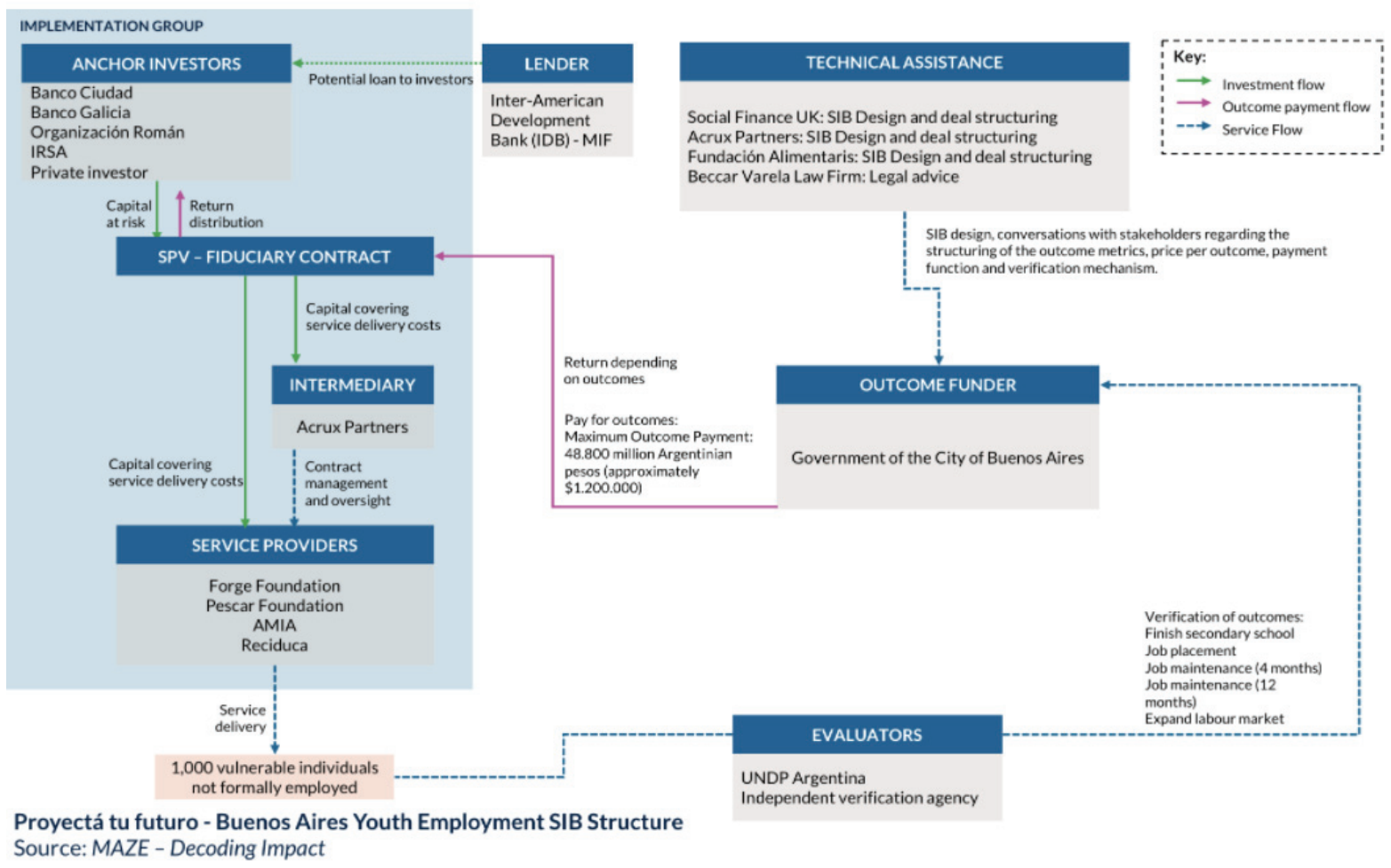


El encuentro entre el sector público y el privado, que caracteriza las diferentes formas de partnership público-privada (PPP), con el objetivo de financiar, construir y gestionar infraestructuras o prestar servicios de interés público, puede ser, en el actual contexto de emergencia del covid-19, una poderosa palanca para la revitalización económica de un país, así como un volante para implementar procesos de desarrollo y renovación.

Las arquitecturas financieras más sofisticadas utilizan un esquema de financiación mixta (blended finance) con la participación de diferentes inversores privados, con diferentes orientaciones hacia la sostenibilidad, y que son, por tanto, portadores de diferentes necesidades de rendimiento e impacto. La mezcla de inversores permite alcanzar importantes cantidades de financiación y asegurar, al mismo tiempo, un bajo costo de financiación.

Desde el punto de vista de la Administración Pública, el uso de la PPP basada en modelos de pago por resultados (pay by results) puede generar ventajas en términos: i) financieros, ya que la inversión se ejecuta sin generar desembolsos de efectivo; ii) económicos, debido al posible ahorro de costes (netos de los costes del proyecto y de la remuneración del inversor); iii) de sostenibilidad ỳ, en relación con el objetivo de impacto del proyecto.

Los beneficios que se derivan de la presencia simultánea de los sectores público y privado en las inversiones no se limitan únicamente al componente financiero; de hecho, las ganancias de eficiencia alcanzables están relacionadas con la posibilidad de reducir el tiempo necesario para ejecutar un programa de inversión, el reparto de riesgos que implica una optimización de los procesos y el mayor nivel de experiencia presente en el proyecto en términos de evaluaciones y organización (Tribunal de Cuentas Europeo, 2018).

La Agenda 2030, y los ODS, son el principio inspirador de la financiación sostenible. En esta dirección, resulta correcto hablar de "finanzas para el desarrollo sostenible" como aquellas finanzas que pretenden dirigir los recursos financieros públicos y privados hacia sectores, proyectos e iniciativas funcionales a la transición de la economía hacia modelos más sostenibles, es decir, más inclusivos y con menor impacto sobre el medio ambiente (ASViS, 2020). Los ODS ayudan a responder a dos preguntas: "qué financiar" y "cómo financiar".

La integración entre los ODS, las PPP y los modelos de pay by results permite a las AP optimizar el apalancamiento financiero al servicio de la sostenibilidad y representa una oportunidad para las AP que están llamadas a seleccionar intervenciones coherentes con sus políticas de desarrollo, al tiempo que tienen que hacer frente a la escasez de recursos. Además, el modelo de pay by results, a través de la implementación de PPP, permite la construcción de redes para la transición hacia el desarrollo sostenible, garantizando la participación activa de las instituciones públicas, los inversores institucionales, la sociedad civil, las entidades del tercer sector y los inversores minoristas. 


\section{MAPA DE PROYECTOS DE FINANCIACIÓN DE IMPACTO EN ESPAÑA Y LATINOAMÉRICA}

El conjunto de acciones/proyectos que a través de los recursos adecuados persiguen el desarrollo sostenible suelen denominarse "buenas prácticas", definidas por la Dirección General de Medio Ambiente de la Unión Europea como "aquellas acciones, exportables a otras realidades, que permiten a un Municipio, a una comunidad o a cualquier Administración Local, avanzar hacia formas de gestión sostenible a nivel local" y por tanto capaces de no comprometer a las generaciones futuras (UNCED, 1987).

A continuación, proponemos un mapeo de "buenas prácticas" de proyectos de finanzas sostenibles inspirados en los ODS, y financiados con arquitecturas financieras construidas sobre formas de pago por resultados de asociación público-privada implementadas, en España y países de América Latina (Tabla 2). En concreto, utilizando también la base de datos del Global Steering Group (GSG) for Impact Investment, ha sido posible clasificar los proyectos de acuerdo con los siguientes criterios:

- el nivel de intervención, explicita el nivel de la AP implicada en la asociación público-privada. El nivel es nacional cuando interviene un organismo del gobierno central, como un Ministerio; regional, cuando interviene un grupo de centros urbanos; municipal, cuando interviene un municipio o un solo centro urbano. En particular, el mapeo identifica el nivel geográfico en el que se sentirá el impacto del proyecto;

- el área de impacto, que identifica las variaciones en los indicadores que constituyen los ODS y las metas cuantitativas;

- la arquitectura financiera, que aclara cómo se financian los proyectos. Se identifican los instrumentos financieros que responden a la lógica de la financiación de impacto, como los bonos de impacto social, los bonos verdes (Cuadro 1), los fondos sociales o los fondos verdes (Cuadro 3);

- los sujetos implicados, es decir, el conjunto de gobiernos locales, entidades territoriales, asociaciones, empresas y ciudadanos que, inicialmente o posteriormente, colaboraron en el proyecto;

- las especificidades del proyecto, en cuanto a objetivos, características y métodos de ejecución. 
Tabla 2: Mapeo de proyectos de financiación sostenible en AP en España y LATAM

\begin{tabular}{|c|c|c|c|c|c|c|}
\hline País & $\begin{array}{c}\text { Estrategia } \\
\text { de } \\
\text { financiación }\end{array}$ & $\begin{array}{c}\text { Nivel } \\
\text { de } \\
\text { intervención }\end{array}$ & Descripción & ODS & $\begin{array}{c}\text { Área de } \\
\text { intervención } \\
\text { (Adl) }\end{array}$ & Descripción Adl \\
\hline España & $\begin{array}{l}\text { Bono } \\
\text { Verde }\end{array}$ & Gobierno & $\begin{array}{l}\text { El Instituto de Crédito Oficial } \\
\text { (ICO) lanzó en } 2019 \text { la } \\
\text { emisión inaugural de Bonos } \\
\text { Verdes por un importe de } \\
500 \text { millones de euros }\end{array}$ & $\begin{array}{l}\text { \#7 Energía } \\
\text { limpia y } \\
\text { accesible } \\
\text { \#13 Lucha } \\
\text { contra el cambio } \\
\text { climático }\end{array}$ & $\begin{array}{l}\text { Ambiente- } \\
\text { Energía } \\
\text { renovable }\end{array}$ & $\begin{array}{l}\text { Se financiarán proyectos } \\
\text { relacionados con la energía } \\
\text { renovable, la eficiencia } \\
\text { energética, el transporte limpio, } \\
\text { la prevención y el control de } \\
\text { la contaminación, la gestión } \\
\text { sostenible de los recursos } \\
\text { naturales y el uso del suelo, y la } \\
\text { gestión sostenible del agua. }\end{array}$ \\
\hline Argentina & $\begin{array}{l}\text { Fondo } \\
\text { Social }\end{array}$ & Gobierno & $\begin{array}{l}\text { EI Ministerio de Producción } \\
\text { ha lanzado un fondo de } \\
\text { capital riesgo llamado } \\
\text { FONDECE. Utiliza criterios } \\
\text { ESG para su estrategia de } \\
\text { selección de inversiones y ha } \\
\text { abierto créditos blandos para } \\
\text { emprendedores de impacto. }\end{array}$ & $\begin{array}{l}\text { \#9 Empresa, } \\
\text { Innovación e } \\
\text { Infraestructura }\end{array}$ & $\begin{array}{l}\text { Social- } \\
\text { Comercio } \\
\text { Empresa }\end{array}$ & $\begin{array}{l}\text { Desarrollo económico local, } \\
\text { cadenas de valor, comercio justo, } \\
\text { inclusión financiera y agricultura. }\end{array}$ \\
\hline
\end{tabular}




\begin{tabular}{|c|c|c|c|c|c|c|}
\hline País & $\begin{array}{c}\text { Estrategia } \\
\text { de } \\
\text { financiación }\end{array}$ & $\begin{array}{c}\text { Nivel } \\
\text { de } \\
\text { intervención }\end{array}$ & Descripción & ODS & $\begin{array}{c}\text { Área de } \\
\text { intervención } \\
\text { (Adl) }\end{array}$ & Descripción Adl \\
\hline Brasil & $\begin{array}{l}\text { Green } \\
\text { Fund }\end{array}$ & Municipio & $\begin{array}{l}\text { El BNDES es el gestor del } \\
\text { Fondo Amazónico (Fundo } \\
\text { Amazônia), creado en } 2008 \\
\text { para recaudar donaciones } \\
\text { destinadas a inversiones } \\
\text { no reembolsables en la } \\
\text { prevención, el seguimiento } \\
\text { y la lucha contra la } \\
\text { deforestación, así como } \\
\text { en la conservación y el uso } \\
\text { sostenible de los bosques } \\
\text { biológicos de la Amazonia. }\end{array}$ & $\begin{array}{l}\text { \#13 Lucha } \\
\text { contra el cambio } \\
\text { climático }\end{array}$ & Ambiente & $\begin{array}{l}\text { El objetivo principal del Fondo } \\
\text { Amazónico es promover la } \\
\text { protección de este patrimonio } \\
\text { y el desarrollo sostenible de la } \\
\text { zona. Se apoyan proyectos en } \\
\text { áreas como: gestión de bosques } \\
\text { públicos y áreas protegidas; } \\
\text { control y seguimiento, así como } \\
\text { inspección ambiental; gestión } \\
\text { forestal sostenible; y actividades } \\
\text { económicas desarrolladas a partir } \\
\text { del uso sostenible de la foresta. }\end{array}$ \\
\hline Chile & $\begin{array}{l}\text { Fondo } \\
\text { Social }\end{array}$ & Municipio & $\begin{array}{l}\text { El Fondo de Solidaridad e } \\
\text { Inversión Social (FOSIS), es } \\
\text { un servicio del Gobierno de } \\
\text { Chile, creado el } 26 \text { de octubre } \\
\text { de } 1990 \text {. EI FOSIS tiene } 16 \\
\text { direcciones regionales y } \\
20 \text { oficinas provinciales y } \\
\text { trabaja con organizaciones } \\
\text { locales para poner en marcha } \\
\text { programas de integración. }\end{array}$ & $\begin{array}{l}\text { \#1 Superación } \\
\text { de la pobreza } \\
\text { \#11 Ciudades } \\
\text { y comunidades } \\
\text { sostenibles }\end{array}$ & $\begin{array}{l}\text { Social- } \\
\text { Casa y } \\
\text { Urbanistica }\end{array}$ & $\begin{array}{l}\text { Proporcionar oportunidades a } \\
\text { quienes viven en la pobreza y } \\
\text { la vulnerabilidad social a través } \\
\text { de su autonomía de ingresos, } \\
\text { empoderamiento social, vivienda } \\
\text { y programas medioambientales. } \\
\text { Tratar de mejorar la calidad de } \\
\text { vida de los barrios y localidades } \\
\text { vulnerables mediante el trabajo } \\
\text { en colaboración. }\end{array}$ \\
\hline México & $\begin{array}{l}\text { Bono } \\
\text { Verde }\end{array}$ & Gobierno & $\begin{array}{l}\text { El } 29 \text { de octubre de } 2015 \text {, } \\
\text { Nacional Financiera Banca } \\
\text { de Desarrollo (NAFIN) emitió } \\
\text { un bono verde de } \$ 500 \\
\text { millones. }\end{array}$ & $\begin{array}{l}\text { \#7 Energía } \\
\text { limpia y } \\
\text { asequible }\end{array}$ & $\begin{array}{l}\text { Ambiente- } \\
\text { Energia } \\
\text { renovable }\end{array}$ & $\begin{array}{l}\text { Los ingresos netos de la emisión } \\
\text { de los pagarés se utilizan para } \\
\text { financiar proyectos de generación } \\
\text { de energía eólica elegibles en } \\
\text { México }\end{array}$ \\
\hline
\end{tabular}

El mapeo destaca algunos rasgos distintivos y las principales orientaciones que han caracterizado las experiencias de financiación sostenible y asociación público-privada de las AP en las áreas observadas.

En general, los proyectos prefieren algunas áreas de impacto: trabajo, innovación energética y de infraestructuras, lucha contra el cambio climático. Las inversiones en proyectos sociales y medioambientales se financian principalmente con fondos (Cuadro 3). 


\section{CUADRO 3 - FONDO SOCIAL Y FONDO VERDE}

Una posible clasificación distingue los fondos socialmente responsables de los fondos ESG y los fondos de impacto. Los primeros invierten en empresas cotizadas en los mercados financieros, aplicando técnicas de selección: positiva, negativa, mixta, best in class; los fondos ESG invierten en empresas cotizadas o no cotizadas siguiendo una actitud más activa basada en tres enfoques principales: selección, temática, best in class, integración ESG, shareholder advocacy. Los fondos de impacto invierten en empresas o proyectos siguiendo tres enfoques principales (temático, integración ESG, advocacy) aplicando el modelo pay by results para atraer una mezcla de diferentes inversores.

Mientras los Bonos de Impacto Social, son una arquitectura financiera que se basa sobre un modelo de acuerdo público-privado (public private partnership) que no requiere una emisión en el mercado.

En particular, en el área observada, hay tres fondos sociales activos en Argentina, Brasil y Chile, respectivamente a nivel de Gobierno, Región y Municipio; en Brasil, también hay un fondo verde activo a nivel de Municipio. Los objetivos que persiguen los fondos están relacionados con el comercio, la vivienda y el urbanismo, el empleo y el medio ambiente (Tabla 3).

Tabla 3: Highlights de Mapeo

\begin{tabular}{|l|c|c|c|c|}
\hline \multicolumn{2}{|c|}{ Arquitectura financiera } & \multicolumn{3}{c|}{ Nivel de intervención Área de intervención (ODS) } \\
\hline Gobierno & Región & Municipio & \\
\hline Bono de impacto social & & & 1 & \\
\hline Bonos verdes & 2 & 1 & & \\
\hline Fondos & 1 & 1 & 2 & \\
\hline
\end{tabular}

Fuente: elaboración propia

\section{PERSPECTIVA FUTURA}

La transición hacia el crecimiento sostenible sólo puede comenzar desde el territorio; en esta perspectiva, el papel de la AP se vuelve crucial. Y, sin embargo, es precisamente la AP la que está más retrasada en la implementación de vías de sostenibilidad que sean capaces de combinar los ODS con políticas presupuestarias y de gasto público coherentes.

Según un enfoque programático de la sostenibilidad, las AP deben ser capaces de responder a varias preguntas: ¿Cuáles son las urgencias del contexto local de referencia? ¿A qué áreas del bienestar de las personas se pueden referir? ¿Qué recursos se destinan a las distintas dimensiones del bienestar? ¿Son estas urgencias propias del territorio o están extendidas también en otros contextos? ¿Qué servicios y/o proyectos se han llevado a cabo? ¿Con qué resultados e impactos observables y medibles? ¿Es posible activar sinergias entre diferentes áreas administrativas, generando efectos multiplicadores en términos de inversiones e impacto directo-indirecto? 
Para responder a estas preguntas, las AP deben dotarse de modelos capaces de planificar y supervisar las opciones de inversión en función de estos objetivos. La literatura todavía ofrece pocas referencias a este respecto (La Torre et al., 2020) y, sobre todo, las experiencias aplicadas de los modelos teóricos se encuentran, esencialmente, en una fase prematura.

Las políticas europeas dedicadas a la sostenibilidad serán sin duda un importante y, al mismo tiempo, imprescindible campo de pruebas; el escenario prospectivo, sin embargo, mostrará inevitablemente que las distintas AP avanzan a ritmos diferentes, y con distintas capacidades de gestión y administración. Las AP que sean capaces, en primer lugar, de asumir el reto serán también importantes puntos de referencia para aquellas realidades menos resistentes, o para otras que imaginen emprender el camino hacia una transición sostenible simplemente desde la perspectiva del cumplimiento de la normativa nacional y europea.

La perspectiva esbozada en este trabajo, inspirada en la experiencia de la financiación de impacto -capaz de diseñar instrumentos del tipo pay by results-, representa un modelo para acompañar a la AP hacia nuevos sistemas de gestión de la relación entre la oferta y la demanda de servicios. Analizar las necesidades del territorio en términos de ODS, construyendo vías de impacto para su resolución, atrayendo recursos de inversores orientados al impacto, permite poner la financiación pública al servicio del desarrollo sostenible, superar el mecanismo de asistencialismo de la misma, para moverse en una lógica de retribución ligada al impacto. Otra de las ventajas de integrar en un mismo modelo los ODS, la PPP y las estructuras pay by results es la de construir consenso, participación y responsabilidad alrededor de objetivos comunes, permitiendo que cada institución sea protagonista del cambio: la AP que orienta la financiación hacia la sostenibilidad, los inversores privados que apoyan la intencionalidad del impacto sin reunirse con objetivos de lucro, las organizaciones sin ánimo de lucro que llevan a cabo los proyectos necesarios para lograr el impacto, los intermediarios financieros que, cada vez más, se convierten en un eje de canalización de recursos dedicados al desarrollo sostenible.

Las consecuencias de la pandemia, aún en curso, han forzado inevitablemente un cambio de ritmo radical en el tratamiento de las cuestiones relacionadas con las políticas de desarrollo sostenible, que hoy se sitúan y se situarán cada vez más en el centro de la agenda política, así como en las opciones de producción, inversión, financiación y consumo. La evolución reciente del marco normativo y de la política europea retoma lo que ya está ampliamente recogido en la Agenda 2030. Los responsables políticos tienen la tarea de identificar las prioridades con las que diseñar las estrategias de inversión, mientras que otros actores del sistema económico tienen la tarea de cofinanciar y ejecutar los proyectos.

El modelo propuesto en este trabajo puede ser útil para diseñar una nueva fase en el ciclo de vida de la administración pública, fuertemente caracterizada por una orientación hacia la sostenibilidad ambiental y social. La adopción de modelos financieros como el pay by results y la financiación mixta por parte de la AP, se conjugan bien con el proyecto político europeo y con el Objetivo 17 de la Agenda 2030 -reforzar los medios de ejecución y renovar la asociación mundial para el desarrollo sostenible- y favorece la adopción de un enfoque ascendente que contempla el desarrollo sostenible según los términos de una lógica glocal que potencia la AP en sus diferentes articulaciones territoriales. 


\section{BIBLIOGRAFÍA Y SITOGRAFÍA}

- Amazon Fund. (2008). Extraído de https://www.bndes.gov.br/SiteBNDES/bndes/bndes_en/Institucional/ Social_and_Environmental_Responsibility/amazon_fund.html

- Bono verde CDMX. (2018). Extraido de http://procesos.finanzas.cdmx.gob.mx/bono_verde/

- Corte dei Conti Europea. (2018). Partenariati pubblico-privato nell'UE: carenze diffuse e benefici limitati. Relazione speciale N.9. Extraido de: https://op.europa.eu/webpub/eca/special-reports/ppp-9-2018/it/

- Department of Economic and Social Affairs, United Nations. SDG Indicators. Extraido de: https://unstats. un.org/sdgs/indicators/indicators-list/

- European Commission. (2018). Action Plan: Financing Sustainable Growth. Brussels, Belgium

- European Commission (2020). Recovery Plan Europe. Extraido de: https://ec.europa.eu/info/strategy/ recovery-plan-europe_it

- Finanza per lo sviluppo sostenibile: un tema strategico per l'agenda 2030 (2020). ASviS. Extraido de: https://asvis.it/public/asvis2/files/Approfondimenti/GdL_Trasv_FINANZA.pdf

- Forum Nazionale per lo Sviluppo Sostenibile. Extraido de https://www.minambiente.it/pagina/verso-laconferenza-nazionale-lo-sviluppo-sostenibile

- Good in Finance. Extraido de https://www.goodinfinance.com/

- Global sustainable investment alliance (2018). Global sustainable investment Review

- ICO launches its first green bonds issue and reaffirms its status as one of the main issuers of sustainable bonds on the European market. (2019). Extraido de https://www.ico.es/web/ico_en/press-release/-/ blogs/ico-launches-its-first-green-bonds-issue-and-reaffirms-its-status-as-one-of-the-main-issuers-ofsustainable-bonds-on-the-european-market

- La Torre M., (2017), Inversiones de Impacto Social: Que Camino para la Union Europea?. Economistas sin Frontieras. N. 27.

- La Torre M., Semplici L, Salazar Zapata J. D. (2020). Un modello di impact finance per i comuni: il piano strategico di mandato BES-oriented. Corporate Governance and Research \& Development Studies. N.2/2020, 144-170.

- Localising the Sustainable Development Goals (2020). Joint Research Center, European Commission. Extraido de: https://urban.jrc.ec.europa.eu/sdgs/en/

- NAB countries (2019). GSG Driving real impact. Extraido de: https://gsgii.org/nab-countries/

- Nafin's annual green bond report. (2018). Extraido de https://www.nafin.com/portalnf/files/secciones/ piso_financiero/pdf/relacion_con_inversionistas/green_bond/Reporte_Nafin_201810.pdf

- OECD. (2019). Social Impact Investment 2019: The Impact Imperative for Sustainable Development. Paris: OECD Publishing.

- Programas Fondo de Solidaridad e Inversion Social. (2020). Extraido de https://www.fosis.gob.cl/ es/programas/

- Proyectá tu futuro - Buenos Aires Youth Employment Social Impact Bond (2018). Government Outcome Labs, Oxford University. Extraido de: https://golab.bsg.ox.ac.uk/knowledge-bank/case-studies/ improving-employability-buenos-aires/

- Stockholm Resilience Centre, Stockholm University (2016). 2030 Agenda HLPF report 
- Support for social and environmental projects. (2020). Extraido de https://www.bndes.gov.br/ SiteBNDES/bndes/bndes_en/Institucional/Social_and_Environmental_Responsibility/support_for_ social_environmental_projects.html

- The Social Bond Principles (2020). International Capital Market Association (ICMA). Extraido de: https://www.icmagroup.org/sustainable-finance/the-principles-guidelines-and-handbooks/social-bondprinciples-sbp/

- United Nations. (1987). Our common future. Report of the World Commission on Environment and Development. 